\title{
Recognition of Depression in Older Medical Inpatients
}

\author{
Monica Cepoiu, MD, MSc ${ }^{7}$, Jane McCusker, MD, DrPH ${ }^{1,2}$, Martin G. Cole, $F R C P(C)^{3,4}$, \\ Maida Sewitch, $P h D^{1,5}$, and Antonio Ciampi, $P h D^{1,2}$
}

\begin{abstract}
'Department of Clinical Epidemiology and Community Studies, St. Mary's Hospital, 3830 Lacombe Avenue, Montreal, QC H3T 1M5, Canada; ${ }^{2}$ Department of Epidemiology and Biostatistics, McGill University, Montreal, QC, Canada; ${ }^{3}$ Department of Psychiatry, St. Mary's Hospital, Montreal, QC, Canada; ${ }^{4}$ Department of Psychiatry, McGill University, Montreal, QC, Canada; ${ }^{5}$ Department of Medicine, St. Mary's Hospital, Montreal, QC, Canada.
\end{abstract}

BACKGROUND: Studies of recognition of depression in older (aged 65 or more) medical inpatients show low rates of recognition of depression by attending physicians. However, few studies have compared different measures of recognition of depression.

OBJECTIVES: (1) To compare the validity of four indicators of recognition of depression and a global measure of recognition against a diagnosis of depression and (2) to explore the effect of patient characteristics on recognition of depression.

METHODS: In a cohort of 264 medical inpatients 65 years and older ( 115 with major or minor depression, 78 with no depression), sensitivities, specificities, and diagnostic odds ratios (DOR) of 4 indicators of recognition (symptoms, diagnosis, treatment, and referral) and a global measure of recognition (any of the 4 indicators) were calculated. The associations between patient characteristics (age, sex, history of depression, antidepressant use before admission, severity of depression, comorbidity, duration of hospitalization, disability, and hospital of admission) and recognition were explored using multiple logistic regression.

RESULTS: Less than half of the depressed patients were recognized. The indicator with the highest sensitivity was treatment $(27.8 \%, 95 \%$ confidence interval [CI] 20.0-37.0), whereas the indicator with the best specificity was diagnosis (96.6\%, 95\% CI 91.9-98.7). The unadjusted DOR of global recognition was 2.6 (95\% CI 1.5, 4.4). Less comorbidity, more severe depression symptoms, a history of depression, longer hospital stay, and antidepressant use before admission were significantly associated with better global recognition.

CONCLUSION: Recognition of depression in elderly medical inpatients depends upon the indicator of recognition used.

KEY WORDS: recognition; depression; inpatients.

DOI: $10.1007 / \mathrm{s} 11606-006-0085-0$

(C) 2007 Society of General Internal Medicine 2007;22:559-564

Published online January 11, 2007

\section{INTRODUCTION}

Depression is a mood disorder with a high prevalence in seniors (defined as those aged 65 years and older). Up to $16.5 \%$ of seniors in the community ${ }^{1-3}$ and $29 \%$ of those in primary care ${ }^{4}$ have depression, diagnosed either by a structured clinical interview or a depression scale. In hospitalized seniors, the prevalence of major depression varies from $10 \%$ to $44.5 \%$, and that of minor depression from $7.9 \%$ to $25 \%{ }^{5,6}$ Late life depression is associated with an increased use of medical resources, ${ }^{7-10}$ decreased compliance with prescription medication for a chronic illness, ${ }^{11}$ and increased mortality. ${ }^{12,13}$ Depressive symptoms associated with poor physical health lead to an increase in health care costs. ${ }^{7,9,10,14}$

In the literature, the term recognition of depression indicates that a clinical diagnosis of depression was made by a health professional, without using rating instruments or structured clinical interviews. A clinical diagnosis of depression may be ascertained by review of patient charts and/or discharge notes, by looking for depression diagnoses and/or antidepressant treatment in medical databases, or by simple questions addressed to physicians regarding a possible diagnosis of depression. ${ }^{15-19}$ Studies assessing the recognition of depression by physicians report that the sensitivity of recognition (i.e., the proportion of depressed seniors who are recognized as depressed) varies in seniors between $8.7 \%$ in elderly medical inpatients ${ }^{19}$ and $67 \%$ in primary care patients. ${ }^{20}$ Less frequently assessed are the specificity of recognition (i.e., the proportion of nondepressed seniors correctly identified as not depressed), which varies between $75 \%$ and $91.5 \%,{ }^{18,21}$ the positive predictive value of recognition (32\%), ${ }^{21}$ and kappa of agreement between physician recognition and the gold standard diagnosis of depression (0.31 and 0.29). ${ }^{18,22}$

Sources of variability in recognition rates reported in the literature include the measure of recognition and the gold standard diagnosis of depression. Recognition may be ascertained by chart review or by physicians report, the chart review yielding a lower sensitivity of recognition than physician report. ${ }^{20,23}$ When chart review is the method of assessment, recognition of depression is indicated by $1,2,3$, or all 4 of the following: diagnosis of depression, notes of depressive symptoms, treatment of depression, and referral to psychiatrist. $^{20,21,23-29}$ As gold standard diagnosis of depression, some studies used a structured clinical interview administered by research staff, ${ }^{19,25,30}$ whereas other studies ${ }^{17,24}$ used rating 
instruments with specific cut points administered by research staff or self-completed by patients.

Fewer studies have examined the factors (patient or physician) associated with recognition of depression. Factors associated with increased recognition of depression include female sex, age over 65, white, Hispanic, or Asian ethnicity, increased physical disability, and increased severity of depression. ${ }^{16-18,24,29,30,31,33,34}$

The present study aims to compare the validity of 4 indicators and a global measure of recognition in a sample of older medical inpatients, using a gold standard research diagnosis of major or minor depression. Also, we explore the influence on recognition of depression of patient characteristics, including sex, age, severity of depressive symptoms, prior antidepressant use, and a history of depression.

\section{METHODS}

The present study is a cross-sectional secondary analysis of data collected for 2 studies conducted in two Montréal hospitals: a randomized controlled trial (RCT) of systematic detection and multidisciplinary care of major depression ${ }^{35}$ and an observational prospective study that compared outcomes in patients with and without depression. ${ }^{33}$ These studies were concurrent and used the same data collection staff and measures. For both studies, the sampling frame consisted of patients aged 65 and older recruited from the emergency department to the medical services. Patients admitted to the intensive care unit or cardiac monitoring unit for more than 48 hours, with imminently terminal illness, who did not speak or understand English or French, did not live on the island of Montreal (to facilitate follow-up), or with more than mild cognitive impairment were excluded in both studies. Details on recruitment and screening methods are presented elsewhere. $^{33,35}$

Among the 1,686 eligible patients, 219 patients with current major depression, 137 with current minor depression, and a random sample of 186 with no depression according to the Diagnostic Interview Schedule (DIS) were enrolled in the prognosis study. Diagnostic Interview Schedule was administered at enrolment (T1) by a trained clinical assistant and during the hospitalization or shortly after discharge (T2) by a research assistant (RA). Seventy-eight patients in the intervention arm of the RCT were excluded from this analysis as they were all seen by a psychiatrist, as part of the intervention tested; 79 patients in the control arm were included because treating physicians were not informed of the research diagnosis of depression and these patients received usual hospital care. Furthermore, patients without the DIS completed by the RA or with the DIS completed by the RA more than a week after discharge were excluded. The final sample size of the present study was 264 .

\section{Measurements}

Data were collected by trained RAs and were derived from patient interviews, from chart review, from hospital administrative databases, and from the prescription database of Régie de l'assurance maladie du Québec (RAMQ), which provides universal insurance for medications for the Quebec population aged 65 years or older. The research personnel collecting the data was blinded to patients' screening diagnosis.

The term recognized used in this study implies that the patient was considered depressed by the attending physician, even if the patient had no diagnosis of major or minor depression according to DIS completed at T1 and T2. Four indicators of recognition were defined (recognition by diagnosis, symptoms, treatment, and referral), based on chart review and the RAMQ database, to cover all the possible methods of documentation of depression by the attending physician. Recognition by diagnosis indicates the presence of one or more of the following in the attending physician progress notes: major depression, minor depression, dysthymic disorder, or adjustment disorder. Recognition by symptoms indicates the presence of any of the following symptoms in the progress notes: depression, depressed, depressive mood, sad, crying, decreased mood, guilt, discouraged, unhappy, down mood, down, in the dumps, low, miserable, or tearful. These words are similar to those describing the mood in a Major Depressive Episode according to DSM-IV. ${ }^{36}$ Recognition by treatment was based on information obtained from the prescription database and defined as the presence of an antidepressant prescription in the 2 months after the discharge date of the index hospitalization. Data regarding prescription medications received during hospitalization were not available. Recognition by referral indicates the presence of a recommendation for a psychiatric consultation made in the chart by the attending physician. A global measure of recognition (global recognition) was defined as the presence of any 1 of the 4 indicators of recognition.

A gold standard diagnosis of depression by the DIS (based on DSM-IV criteria for current major and minor depression) ${ }^{37}$ was used to evaluate the validity of the indicators of recognition described above. Depression (major or minor) was diagnosed twice, at time 1 (T1) (the day after hospital admission, by a clinical assistant, to identify the study sample) and time 2 (T2) (as soon as possible after T1, by a RA, to collect baseline data for the study). In the present study, the term depressed ( $n=$ 115) was used to indicate that the patient was diagnosed at both $\mathrm{T} 1$ and $\mathrm{T} 2$ with either minor or major depression. We decided to combine major and minor depression based on preliminary analysis that showed no important differences in recognition rates in patients with major depression compared to those with minor depression at T1, and insufficient sample sizes to examine the two diagnoses separately. The patients diagnosed with major or minor depression at only one time (T1 or T2) and patients who were not depressed at both times (T1 and T2) were included in the comparison group of nondepressed patients $(n=149)$.

The physical health status of the patients enrolled in the study was evaluated using the 36-item short-form health survey (SF-36) physical component summary scale questions. ${ }^{38}$ Comorbidity was assessed by Charlson comorbidity index (CCI) based on chart review data on the previous 2 years, ${ }^{39}$ cognitive status by the Mini-Mental State Examination at $\mathrm{T} 2,{ }^{40}$ physical disability by the Older Americans Research Survey activities of daily living (ADL) at $\mathrm{T} 2,{ }^{41}$ and the severity of the present episode of depression by the Hamilton depression scale (HAMD) (21-item version) at T2. ${ }^{42}$ Information regarding the patients' antidepressant use during the 2 months before admission and after discharge was extracted from RAMQ database. 
Table 1. The study groups

\begin{tabular}{lccr}
\hline & Depressed $^{*}$ & Nondepressed $^{\dagger}$ & Total \\
\hline Recognized as depressed $^{\ddagger}$ & $\mathrm{TP}=49$ & $\mathrm{FP}=33$ & 82 \\
Not recognized as depressed $^{\S}$ & $\mathrm{FN}=66$ & $\mathrm{TN}=116$ & 182 \\
Total & 115 & 149 & 264 \\
\hline
\end{tabular}

Calculation of crude sensitivity, specificity, and DOR: sensitivity $=T P /(T P+$ $F N)$; specificity $=T N /(T N+F P)$; and $D O R=(T P / F N) /(F P / T N)=[$ sensitivity $/$ $(1-$ sensitivity $)] /[(1-$ specificity $) /$ specificity]

$T P=$ true positives, $T N=$ true negatives, $F P=$ false positives, $F N=$ false negatives

*Diagnosed with major or minor depression at T1 (at enrolment) and T2 (at baseline)

${ }^{\dagger}$ Diagnosed with major or minor depression at only 1 time (T1 or T2) and no depression at both $\mathrm{T} 1$ and $\mathrm{T} 2$

${ }^{*}$ By 1 or more of the 4 indicators of recognition

${ }^{\mathrm{B}_{B}} \mathrm{I} 1$ or more of the 4 indicators of recognition

\section{Statistical Analyses}

To assess the validity of physicians' recognition of depression, we calculated sensitivities, specificities, and diagnostic odds ratios (DOR) of recognition in the whole sample. Diagnostic odds ratios can be derived from the sensitivity and specificity and is relatively independent of changes in both prevalence and spectrum of the disease. ${ }^{43,44}$ In depressed patients, we used multiple logistic regression to construct models for each indicator of recognition and of global recognition that describes the relation between several patient characteristics and recognition of depression. All statistical analyses were carried out using SAS 9.1 for Windows.

\section{RESULTS}

The sample used in analysis consisted of 264 patients. Among them, 115 (43.6\%) were diagnosed as depressed at both $\mathrm{T} 1$ and $\mathrm{T} 2$ and $82(31.1 \%)$ were recognized as depressed by 1 or more of the 4 indicators of depression (Table 1). Demographic and clinical characteristics of the patients are presented in Table 2. The average age of the subjects was 79.5 years $(\mathrm{SD}=7.0)$ and the majority were women and had premorbid ADL disability.

Sensitivities, specificities, and DOR for all 4 indicators of recognition and the global measure of recognition are presented in Table 3. Among the 4 indicators, recognition by treatment had the highest sensitivity, but the lowest specificity and DOR. The indicator with the highest accuracy, as indicated by DOR, was recognition by diagnosis $(3.6,95 \%$ confidence interval [CI] 1.2-10.6).

Table 4 presents the odds of recognition in the sample of 115 depressed patients by SF-36 score, CCI, history of depression, HAMD score, duration of hospitalization, antidepressant use before admission, and hospital of admission. Patients with low comorbidity $(\mathrm{CCI}<1)$ and long duration of hospitalization ( $\geq 9$ days) had significantly higher odds $(P<.05)$ of being recognized as depressed by diagnosis. Recognition by symptoms was significantly increased $(P<.05)$ only in patients with long duration of hospitalization ( $>9$ days). Recognition by treatment was significantly increased in patients with a history of depression, high severity of depression, and antidepressant use before admission. Recognition by referral was significantly higher in patients with high SF-36 scores (>35.2), a history of depression, high severity of depression, long duration of hospitalization, or admitted to one of the hospitals (hospital A). The final model of global recognition included 6 covariates, all statistically significant $(P<.05)$. Patients with low comorbidity $(\mathrm{CCI}<1)$, a history of depression, high severity of depression (HAMD scores $>18$ ), long duration of hospitalization ( $>9$ days), antidepressant use before admission, and admission to hospital A had increased odds of being recognized as depressed.

\section{DISCUSSION}

To our knowledge, this is the first study to compare the validity of 4 indicators of physician recognition of depression (recognition by diagnosis, symptoms, treatment, and referral). The sensitivity of global recognition was low $(42.6 \%$, 95\% CI 33.552.1); more than half of older medical inpatients with major or minor depression were not recognized by attending physicians by any of the 4 indicators. The specificity of global recognition found in our study $(77.8 \%, 95 \%$ CI $70.1-84.0)$ suggests that physicians correctly identified as not depressed about 3 of 4 patients. The odds of being recognized as depressed by physicians were 2.6 times higher in depressed versus not depressed patients. The low sensitivities of recognition by symptoms and diagnosis suggest that documentation of depression by physicians in patients' charts is poor. Thus, studies that rely only on chart review report the lowest sensitivities of recognition. ${ }^{19,23,45}$ Also, the fact that recognition by treatment had the lowest accuracy (lowest DOR) indicates that older medical inpatients may not receive the appropriate treatment for depression.

Table 2. Demographic and clinical characteristics of the sample

\begin{tabular}{ll}
\hline \multicolumn{1}{c}{ Variables } & Total sample (N=264) \\
\hline Age [mean (SD)] & $79.5(7.0)$ \\
Male [n (\%)] & $105(39.8)$ \\
First language [n (\%)] & \\
English & $83(31.5)$ \\
French & $45(17.0)$ \\
Other & $136(51.5)$ \\
Education [n (\%)] & \\
O-6 years & $30(11.4)$ \\
$7-11$ years & $99(37.5)$ \\
12 or more years & $135(51.1)$ \\
Marital status [n (\%)] & \\
Married & $82(31.0)$ \\
Single & $25(9.5)$ \\
Separated/divorced & $27(10.3)$ \\
Widowed & $130(49.2)$ \\
SF-36 physical [mean (SD)] & $35.3(10.8)$ \\
Missing & $(31)$ \\
Charlson index [mean (SD)] & $1.6(1.7)$ \\
Premorbid ADL disability [n (\%)] & \\
No & $90(35.2)$ \\
Yes & $167(64.8)$ \\
missing & $(8)$ \\
MMSE [mean (SD)] & $25.6(3.5)$ \\
Missing & $(20)$ \\
HAMD [mean (SD)] & $13.8(7.0)$ \\
Missing & $(8)$ \\
History of depression [n (\%)] & $48(18.2)$ \\
Antidepressant treatment before & $47(17.8)$ \\
admission [n (\%)] & \\
Missing & $14.8(18.9)$ \\
Duration of hospitalization [mean (SD)] & \\
\hline
\end{tabular}

SF-36=36-item short-form health survey, MMSE=Mini-Mental State Examination 
Table 3. Crude sensitivities, specificities, diagnostic odds ratios (DOR), and their $95 \%$ confidence interval (Cl) for each indicator of recognition and the global measure of recognition $(N=264)$

\begin{tabular}{|c|c|c|c|}
\hline Indicator & $\begin{array}{l}\text { Sensitivity }(95 \% \mathrm{Cl}) \text {; depressed }=115 \text {, } \\
\text { not depressed }=149\end{array}$ & $\begin{array}{c}\text { Specificity }(95 \% \mathrm{Cl}) \text {; depressed }=115 \text {, } \\
\text { not depressed }=149\end{array}$ & $\begin{array}{c}\text { DOR }(95 \% \mathrm{Cl}) ; \text { depressed }=115 \text {, } \\
\text { not depressed }=149\end{array}$ \\
\hline Diagnosis* & $11.3(6.3,18.8)$ & $96.6(91.9,98.7)$ & $3.6(1.2,10.6)$ \\
\hline Symptoms ${ }^{\dagger}$ & $11.3(6.3,18.8)$ & $95.9(91.0,98.3)$ & $3.0(1.1,8.2)$ \\
\hline Treatment & $27.8(20.0,37.0)$ & $85.9(79.0,90.8)$ & $2.3(1.2,4.3)$ \\
\hline Referral $^{\S}$ & $23.4(16.2,32.4)$ & $91.9(86.0,95.5)$ & $3.5(1.6,7.2)$ \\
\hline Global recognition ${ }^{\#}$ & $42.6(33.5,52.1)$ & $77.8(70.1,84.0)$ & $2.6(1.5,4.4)$ \\
\hline
\end{tabular}

*Diagnosis of depression (major depression, minor depression, dysthymic disorder, or adjustment disorder) in the attending physician progress note

${ }^{\dagger}$ Emotional symptoms of depression were noted in progress notes.

${ }^{\ddagger}$ An antidepressant prescription in the 2 months after the discharge date of the index hospitalization

${ }^{\S}$ Recommendation for a psychiatric consultation was made in the chart by the attending physician.

\#Any 1 of the 4 indicators of recognition

The sensitivity of global recognition and the DOR in our study were higher than in other studies conducted in medical or surgical wards that were based on chart review only (sensitivities varied between $8.7 \%$ and $32.5 \%)^{19,25,26,46,47}$ Specificity of recognition was lower than those reported in other studies (specificities varied between $84.4 \%$ and 95.2\%). ${ }^{15,18}$ This may be because of the criteria we used to define recognition, which covered all the possible methods of depression documentation in charts. Also, we used a reliable prescriptions database, instead of hospital records, to identify recognition by treatment. The DOR of global recognition in our study was lower than those found in 2 other studies conducted in a similar populations (DOR=5.5 and 4.7 , respectively), ${ }^{46,47}$ but recognition in these 2 studies was ascertained by short questionnaires completed by physicians, a method not used in our study.

In our study, sensitivities of recognition by diagnosis, symptoms, treatment, referral, and global recognition were similar between the 2 age categories (65-79 years and $\geq 80$ years). Sensitivity of recognition by referral was significantly higher in women than in men. All other indicators of recognition and global recognition had similar sensitivities of recognition in the 2 groups, a finding that differs from those of other studies, which report a better recognition in women. ${ }^{24,29,31}$ Nevertheless, a recent study conducted in primary care $^{48}$ found no effect of patients' sex on recognition of depression by the primary care physicians. In our study, more patients with high severity of depression were recognized by physicians, similar to other studies results. ${ }^{16,18,34}$

Sensitivity of recognition by diagnosis was higher in patients with low comorbidity $(\mathrm{CCI}<1)$, which contradicts data from a study conducted in a primary care adult population ${ }^{30}$ reporting a greater sensitivity of recognition in patients with associated diseases (such as diabetes or hypertension). This finding may be because of the fact that primary care patients with associated medical diseases, compared to those without, are seen more frequently by physicians. In our sample of older medical inpatients, the presence of depression in patients with high comorbidity may have been considered of lower significance than other medical diagnoses. Consequently, physicians may have noted less frequently a diagnosis of depression in patients with high comorbidity than in those with low comorbidity.

In our study, moderate to severe disability was associated with high sensitivity of recognition, a similar finding being reported in 2 other studies. ${ }^{16,31}$ Among depressed patients, high severity of depression and history of depression is associated with increased recognition. ${ }^{18}$ The association was confirmed by the higher odds of recognition by treatment, by referral and global recognition in patients with high severity of depression, and a history of depression found in our study. Long duration of hospitalization was associated with increased odds of being recognized by diagnosis, symptoms, referral, and global recognition, a finding that may be because of the greater opportunity to observe depressive symptoms. Another significant factor that influenced the odds of recognition by referral was hospital of admission, which may have been because of different clinical sensitivities to depression in older people at the 2 hospitals in the study.

Several limitations of the present study merit discussion. In the analysis of recognition by treatment, we did not control for disorders other than depression, which can be treated with antidepressants, such as anxiety, myalgia, irritable bowel syndrome, or chronic pain syndrome. ${ }^{49}$ Also, we used chart reviews for documentation of recognition, which may be a less

Table 4. Multivariate logistic regression models in depressed patients $(n=115)$

\begin{tabular}{|c|c|c|c|c|c|}
\hline \multirow[t]{2}{*}{ Variables } & Diagnosis & Symptoms & Treatment & Referral & Global recognition \\
\hline & OR $(95 \% \mathrm{Cl})$ & OR $(95 \% \mathrm{Cl})$ & OR $(95 \% \mathrm{Cl})$ & OR $(95 \% \mathrm{Cl})$ & OR $(95 \% \mathrm{Cl})$ \\
\hline SF-36 score $\geq 35.2$ & & & & $2.3(1.0,5.6)$ & \\
\hline Charlson index $<1$ & $3.2(1.1,9.3)$ & $2.5(0.9,7.1)$ & & $2.0(0.8,4.8)$ & $2.9(1.4,6.2)$ \\
\hline History of depression & & & $3.6(1.1,11.1)$ & $2.7(1.1,6.6)$ & $3.2(1.2,8.4)$ \\
\hline HAMD score $\geq 18$ & $2.2(0.8,6.2)$ & & $4.1(1.3,12.5)$ & $5.6(2.4,13.3)$ & $2.6(1.2,5.5)$ \\
\hline Hospitalization $\geq 9$ days & $4.5(1.2,16.3)$ & $5.8(1.5,22.0)$ & & $3.5(1.4,8.6)$ & $2.8(1.3,5.8)$ \\
\hline Antidepressant used before admission & $2.5(0.8,7.7)$ & & $116.0(33.7,399.2)$ & & $26.0(9.3,72.8)$ \\
\hline Hospital A & & $5.0(0.6,40.2)$ & & $21.4(2.5,181.2)$ & $3.3(1.2,9.3)$ \\
\hline
\end{tabular}

Five multivariate regression models were defined for each indicator of recognition and the global recognition. The odds ratios (ORs) presented are also adjusted for the following patient characteristics: language spoken, country of birth, education, disability, and cognitive status.

$C I=$ confidence interval, $S F-36=36$-item short-form health survey, HAMD = Hamilton depression scale 
complete source of information than physician questionnaires or checklists.

Possible methods of improving recognition of depression in older medical inpatients might include: educational programs for physicians and hospital staff to increase awareness of issues that can hinder diagnosis of depression (such as reluctance of the patient to accept a psychiatric diagnosis or to describe their feelings, or higher comorbidity, which can mask the physical symptoms associated with depression); ${ }^{48}$ designing and implementing educational programs for patients and family members that can increase the acceptance of diagnosis and treatment of depression by older patients; ${ }^{48}$ and designing and implementing management programs for active detection and collaborative treatment of depression in seniors. ${ }^{50}$

Acknowledgements: This study was supported by a grant from Fonds de la recherche en santé du Québec (grant no. 25004-2560) and 2 grants from Institutes for Health Research (grant nos. MOP64462 and 11949).

\section{Potential Conflicts of Interest: None disclosed.}

Corresponding Author: Monica Cepoiu, Department of Clinical Epidemiology and Community Studies, St. Mary's Hospital, 3830 Lacombe Avenue, Montreal, QC H3T 1M5, Canada (e-mail: monica. cepoiu@ssss.gouv.qc.ca).

\section{REFERENCES}

1. Henderson AS, Jorm AF, Mackinnon A, et al. The prevalence of depressive disorders and the distribution of depressive symptoms in later life: a survey using draft ICD-10 and DSM-III-R. Psychol Med. 1993;23(3):719-29.

2. Newman SC, Sheldon CT, Bland RC. Prevalence of depression in an elderly community sample: a comparison of GMS-AGECAT and DSM-IV diagnostic criteria. Psychol Med. 1998;28:1339-45.

3. Beekman ATF, Deeg DJH, Smit JH, van Tilburg W. Predicting the course of depression in the older population: results from a communitybased study in the Netherlands. J Affect Disord. 1995;34:41-9.

4. Van Marwijk H, Hoeksema HL, Hermans J, Kaptein AA, Mulder JD. Prevalence of depressive symptoms and depressive disorder in primary care patients over 65 years of age. Fam Pract. 1994;11(1):80-4.

5. Koenig HG, George LK, Petersen BL, Pieper CF. Depression in medically ill hospitalized older adults: prevalence, characteristics, and course of symptoms according to six diagnostic schemes. Am J Psychiatry. 1997;154(10):1376-83.

6. McCusker J, Cole M, Ciampi A, et al. Twelve-month trajectories of depressive symptoms in older medical inpatients. Am J Geriatr Psychiatry. Submitted.

7. Badger TA. Depression, physical health impairment and service use among older adults. Public Health Nurs. 1998;15(2):136-45.

8. Johnson J, Weissman MM, Klerman GL. Service utilization and social morbidity associated with depressive symptoms in the community. JAMA. 1992;267(11):1478-83.

9. Rapp SR, Parisi SA, Wallace CE. Comorbid psychiatric disorders in elderly medical patients: a 1-year prospective study. J Am Geriatr Soc. 1991;39(2):124-31.

10. Vetter $\mathbf{N}$, Jones $\mathbf{D}$, Victor $\mathbf{C}$. The importance of mental disabilities for the use of services by the elderly. J Psychosom Res. 1982;26 (6):607-12.

11. Ziegelstein RC, Fauerbach JA, Stevens SS, Romanelli J, Richter DP, Bush DE. Patients with depression are less likely to follow recommendations to reduce cardiac risk during recovery from a myocardial infarction. Arch Intern Med. 2000;160(12):1818-1823.

12. Ganzini L, Smith DM, Fenn DS, Lee MA. Depression and mortality in medically ill older adults. J Am Geriatr Soc. 1997;45:307-12.

13. Brown KW, Levy AR, Roserberger Z, Edgar L. Psychological distress and cancer survival: a follow-up 10 years after diagnosis. Psychosom Med. 2003;65:636-43.
14. Cole MG, Bellavance F. Depression in elderly medical inpatients: a meta-analysis of outcomes. Can Med Assoc J. 1997;157(8):1055-60.

15. Meldon Sw, Emerman CL, Schubert DS, Moffa DA, Etheart RG. Depression in geriatric ED patients: prevalence and recognition. Ann Emerg Med. 1997;30(2):141-5.

16. Simon GE, Goldberg D, Tiemens BG, Ustun TB. Outcomes of recognized and unrecognized depression in an international primary care study. Gen Hosp Psychiatry. 1999;21:97-105.

17. Stek ML, Gussekloo J, Beekman AT, van Tilburg W, Westendorp RGJ. Prevalence correlates and recognition of depression in the oldest old: the Leiden 85-plus study. J Affect Disord. 2004;78:193-200.

18. Thompson C, Ostler K, Peveler C, Baker N, Kinmonth A. Dimensional perspective on the recognition of depressive symptoms in primary care. Br J Psychiatry. 2001;179:317-23.

19. Rapp SR, Walsh DA, Parisi SA, Wallace CE. Detecting depression in elderly medical inpatients. J Consult Clin Psychol. 1988;56(4):509-13.

20. McCusker J, Karp E, Yaffe MJ, Cole M, Bellavance F. Determining detection of depression in the elderly by primary care physicians: chart review or questionnaire? Prim Care Psychiatry. 1996;2:217-21.

21. Meldon SW, Emerman CL, Schubert DS. Recognition of depression in geriatric ED patients by emergency physicians. Ann Emerg Med. 1997;30(4):442-7.

22. Tiemens BG, VonKorff M, Lin EH. Diagnosis of depression by primary care physicians versus a structured diagnostic interview. Understanding discordance. Gen Hosp Psychiatry. 1999;21(2):87-96.

23. Pérez-Stable EJ, Miranda J, Muñoz RF, Ying $\mathbf{Y}-\mathbf{W}$. Depression in medical outpatients: underrecognition and misdiagnosis. Arch Intern Med. 1990;150(5): 1083-88.

24. Callahan EJ, Bertakis KD, Rahman A, Helms LJ, Robbins J, Miller J. Depression in primary care: patient factors that influence recognition. Fam Med. 1997;29(3):172-6.

25. Koenig HG, Goli V, Shelp F, Kudler HS, Cohen HJ, Blazer DG. Major depression in hospitalized medically-ill older men: documentation, management, and outcome. Int J Geriatr Psychiatry. 1992;7(1):25-34.

26. Pouget R, Yersin B, Wietlisbach V, Burnand B, Büla CJ. Depressed mood in a cohort of elderly medical inpatients: prevalence, clinical correlates and recognition rate. Aging Clin Exp Res. 2000;12(4):301-7.

27. Rapp SR, Parisi SA, Walsh DA. Psychological dysfunction and physical health among elderly medical inpatients. J Consult Clin Psychol. 1988;56(6):851-5.

28. Whooley MA, Avins AL, Miranda J, Browner Ws. Case-finding instruments for depression: two questions are as good as many. J Gen Intern Med. 1997;12:439-45.

29. Lichtenberg PA, Gibbons AT, Nanna M, Blumenthal F. Physician detection of depression in medically ill elderly. Clin Gerontol. 1993;13 (1):81-90.

30. Borowsky SJ, Rubenstein LV, Meredith LS, Camp P, Jackson-Triche $\mathbf{M}$, Wells KB. Who is at risk of non-detection of mental health problems in primary care? J Gen Intern Med. 2000;15:381-8.

31. Katon WJ, Simon G, Russo J, et al. Quality of depression care in a population-based sample of patients with diabetes and major depression. Med Care. 2004;42(12): 1222-9.

32. Shah A, De T. Depression and anxiety in geriatric inpatients. Documented evidence of depression in medical and nursing case-notes and its implications in acutely ill geriatric inpatients. Int Psychogeriatr. 1998;10 (2): 163-72.

33. McCusker J, Cole M, Dufouil C, et al. The prevalence and correlates of major and minor depression in older medical inpatients. J Am Geriatr Soc. 2005;53(8): 1344-53.

34. Coyne J, Schwenk T, Fechner-Bates S. Non detection of depression by primary care physicians reconsidered. Gen Hosp Psychiatry. 1995; 17:3-12.

35. Cole MG, McCusker J, Elie M, Dendukuri N, Latimer E, Belzile E Systematic detection and multidisciplinary care of depression in older medical inpatients: a randomized trial. Can Med Assoc J. 2006;174 (1):38-44

36. American Psychiatric Association. Practice guideline for major depressive disorder in adults. Am J Psychiatry. 1993;150(suppl 4):1-26.

37. Robins LN, Helzer JE, Croughan J, Ratcliff KS. National Institute of Mental Health Diagnostic Interview Schedule: its history, characteristics, and validity. Arch Gen Psychiatry. 1981;38:381-9.

38. Ware J, Sherbourne C. A 36-item short-form health survey (SF-36). Med Care. 1992;30:286-93.

39. Charlson ME, Pompei P, Ales KL, Mackenzie RC. A new method of classifying prognostic comorbidity in longitudinal studies: development and validation. J Chronic Dis. 1987;40(5):373-83. 
40. Tombaugh TN, McIntyre NJ. The mini-mental state examination: a comprehensive review. J Am Geriatr Soc. 1992;40(9):922-35.

41. Fillenbaum GG. Multidimensional Functional Assessment of Older Adults: The Duke Older Americans Resources and Services Procedures. Lawrence, NJ: Erlbaum Associates Inc; 1988.

42. Hamilton M. Development of a rating scale for primary depressive illness. Br J Soc Clin Psychol. 1967;6:278-96.

43. Glas AS, Lijmer JG, Prins Martin H, Bonsel GJ, Bossuyt PM. The diagnostic odds ratio: a single indicator of test performance. $\mathrm{J}$ Clin Epidemiol. 2003;56:1129-35.

44. Fischer JE, Bachmann LM, Jaeschke R. A readers' guide to the interpretation of diagnostic test properties: clinical example of sepsis. Intensive Care Med. 2003;29:1043-51.

45. Whooley MA, Avins AL, Miranda J, Browner WS. Case-finding instruments for depression. Two questions are as good as many. J Gen Intern Med. 1997; 12:439-45.
46. Sliman RJ, Donohue TA, Jarjoura D, Ognibene AJ. Recognition of depression by internal medicine residents. J Community Health 1992; 17(3): 143-52.

47. Balestrieri M, Bisoffi G, Tansella M, Martucci M, Goldberg D. Identification of depression by medical and surgical general hospital physicians. Gen Hosp Psychiatry. 2002;24(4):11.

48. Katona C, Freeling $\mathbf{P}$, Hinchcliffe $\mathbf{K}$, Blanchard $\mathbf{M}$, Wright $\mathbf{A}$. Recognition and management of depression in late life in general practice: consensus statement. Prim Care Psychiatry. 1995;1:107-13.

49. West Suzanne L, Richter A, Melfi CA, McNutt M, Nennstiel ME, Mauskopf JA. Assessing the Saskatchewan database for outcomes research studies of depression and its treatment. J Clin Epidemiol. 2000;53:823-31.

50. Unutzer J, Katon W, Callahan CM, et al. Collaborative care management of late-life depression in the primary care setting. A randomized controlled trial. JAMA. 2002;288(22):2836-45. 